\title{
A strategy to elucidate the reaction mechanism of electrodes for realistic solid oxide electrochemical cells using a dense bulk material
}

Jinsil Lee ${ }^{\mathrm{a}, \#}$, Minseok Kima,\#, Younki Lee ${ }^{\mathrm{c}}$, Jong Hoon Joo ${ }^{\mathrm{a}, \mathrm{b}, \mathrm{d}}{ }^{*}$

a.Department of Urban, Energy, and Environmental Engineering, Chungbuk National University, 1 Chungdaero, Seowon-gu, Cheongju, Chungbuk 28644, (Republic of Korea)

b.Department of Advanced Material Engineering, Chungbuk National University, 1 Chungdae-ro, Seowon-gu, Cheongju, Chungbuk 28644, (Republic of Korea)

c.Department of Materials Engineering and Convergence Technology, Gyeongsang National University, 501 Jinjudaero, Jinju, Gyeongnam 52828,(Republic of Korea)

\# These authors contributed equally

d. Present address: School of Earth Sciences and Environmental Engineering, Gwangju Institute of Science and Technology, Gwangju, 61005, Republic of Korea 


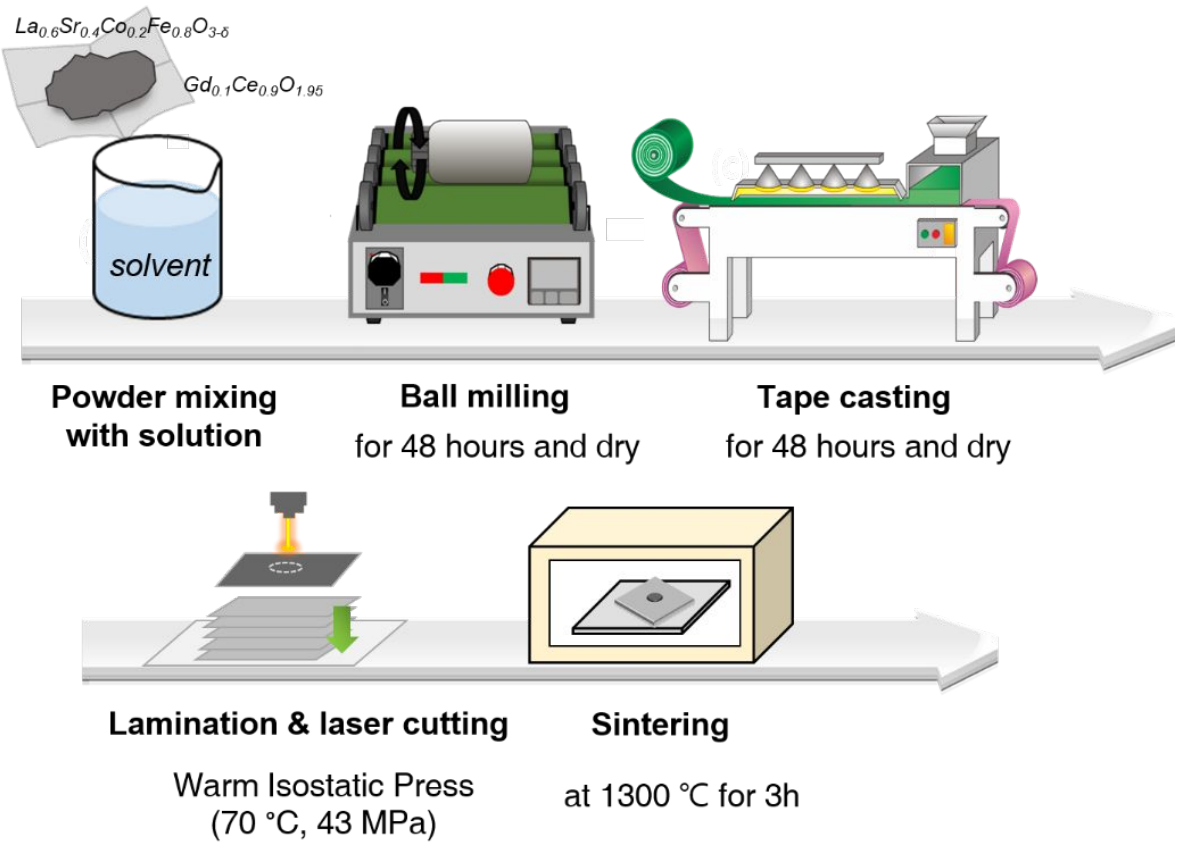

Figure S1. Experimental schematic of manufacturing LSCF dense bulk electrode. 


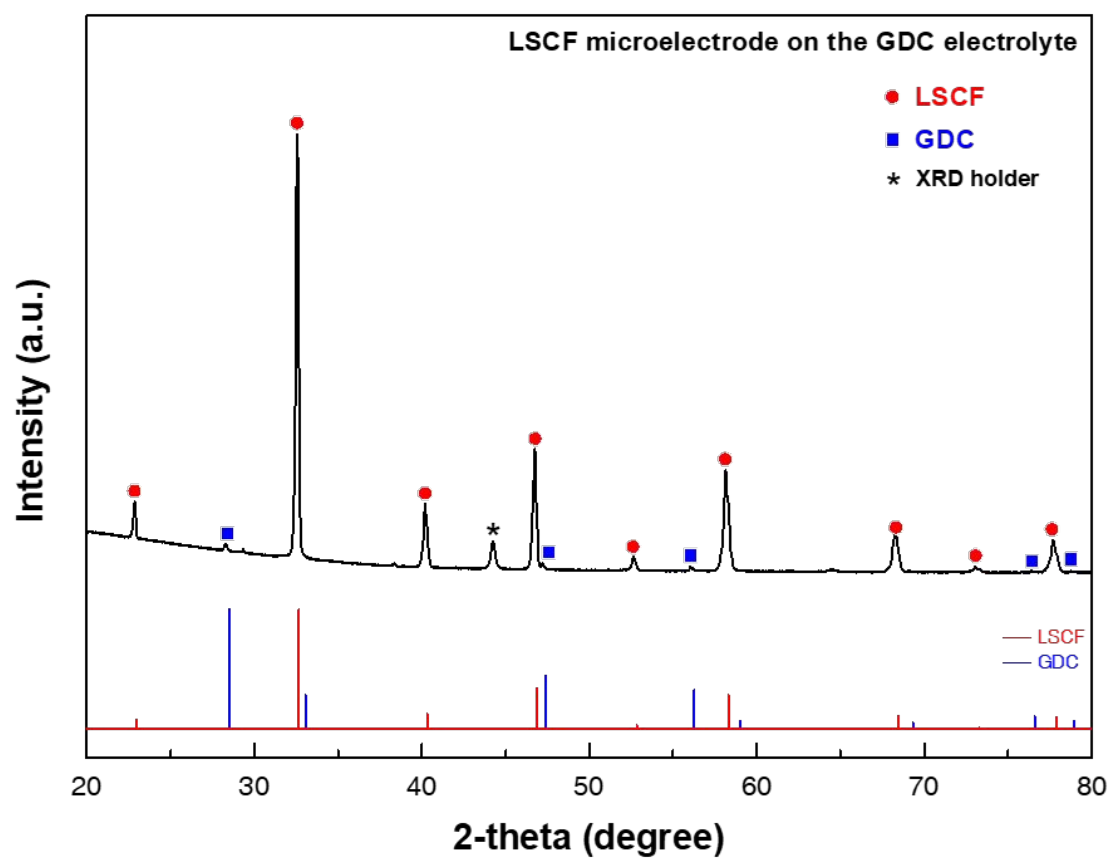

Figure S2. X-ray diffraction pattern of as prepared LSCF thick film on GDC electrolyte at room temperature. All the diffraction peaks correspond to reported patterns. (LSCF was indexed using ICDD 01-079-5623, GDC was indexed using JCPDS \#75-0161) 


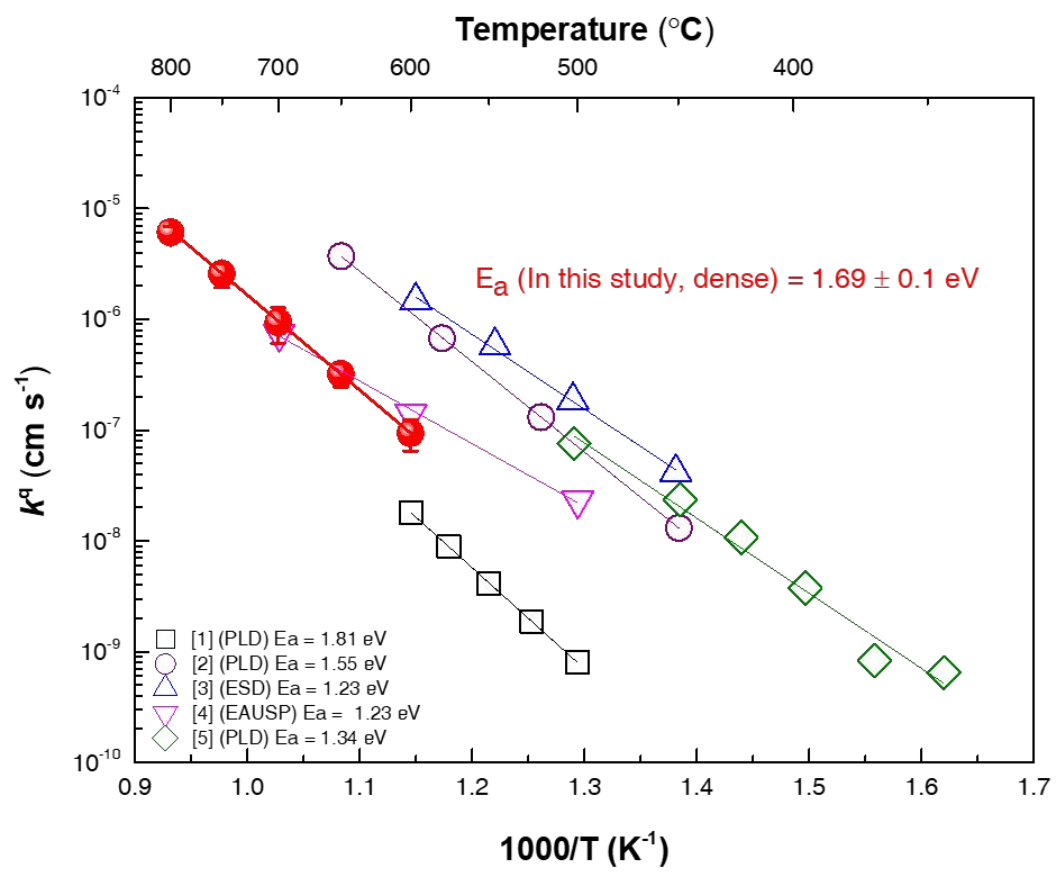

Figure S3. Comparison of temperature dependent surface oxygen exchange kinetics of $\mathrm{La}_{0.6} \mathrm{Sr}_{0.4} \mathrm{Co}_{0.2} \mathrm{Fe}_{0.8} \mathrm{O}_{3}$ according to various the thin film manufacturing methods. (pulsed laser deposition (PLD), electrostatic spray deposition (ESD), electrostatic-assisted ultrasonic spray pyrolysis (EAUSP)). The values of surface oxygen exchange kinetics are range from $10^{-8}$ to $10^{-5} \mathrm{~cm} \mathrm{~s}^{-1}$ at $600^{\circ} \mathrm{C}$. 


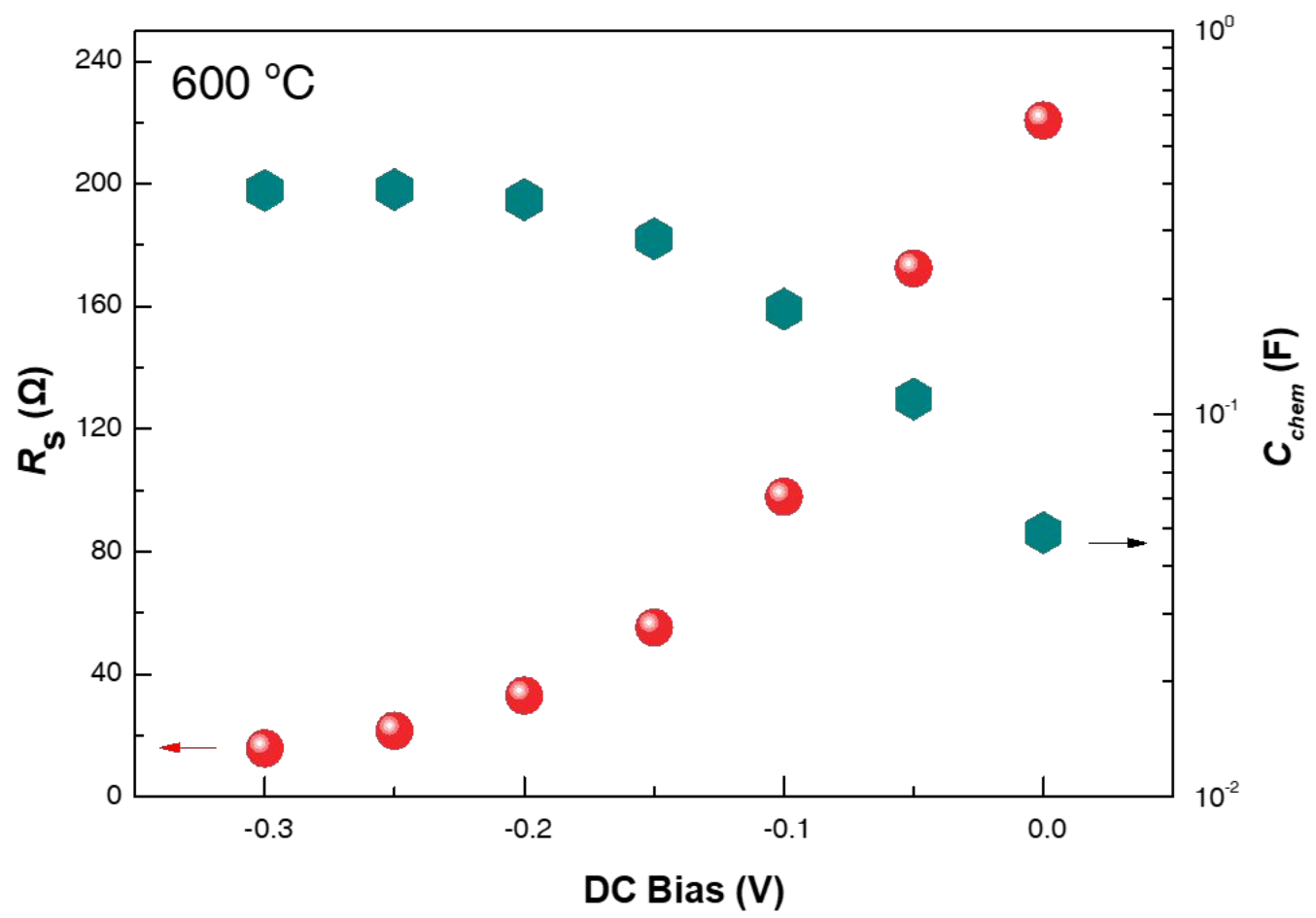

Figure S4. Cathodic dc bias dependence of $R_{s}$ and $C_{\text {chem }}$ range from 0 to $-300 \mathrm{mV}$ at $600{ }^{\circ} \mathrm{C}$.

\section{Reference}


(1) Chen, H.; Guo, Z.; Zhang, L. A.; Li, Y.; Li, F.; Zhang, Y.; Chen, Y.; Wang, X.; Yu, B.; Shi, J. M.; Liu, J.; Yang, C.; Cheng, S.; Chen, Y.; Liu, M.; Improving the Electrocatalytic Activity and Durability of the $\mathrm{La}_{0.6} \mathrm{Sr}_{0.4} \mathrm{Co}_{0.2} \mathrm{Fe}_{0.8} \mathrm{O}_{3-\delta}$ Cathode by Surface Modification. ACS Appl. Mater. Interfaces. 2018, 10, 39785-39793.

(2) Beckel, D.; Muecke, U. P.; Gyger, T.; Florey, G.; Infortuna, A.; Gauckler, L. J.; Electrochemical performance of LSCF based thin film cathodes prepared by spray pyrolysis. Solid State Ion. 2007, 178, 407-415.

(3) Marinha, D.; Dessemond, L.; Djurado, E. Electrochemical investiga-tion of oxygen reduction reaction on $\mathrm{La}_{0.6} \mathrm{Sr}_{0.4} \mathrm{Co}_{0.2} \mathrm{Fe}_{0.8} \mathrm{O}_{3-\delta}$ cathodes deposited by Electrostatic Spray Deposition. J. Power Sources. 2012, 197, 80-87.

(4) Hsu, C. S.; Hwang, B. H.; Microstructure and properties of the $\mathrm{La}_{0.6} \mathrm{Sr}_{0 .{ }_{4}} \mathrm{Co}_{0 .}{ }_{2} \mathrm{Fe}_{0.8} \mathrm{O}_{3}$ cathodes prepared by electrostatic-assisted ultrasonic spray pyrolysis method. $J$. Electrochem. Soc. 2006, 153, A1478-A1483.

(5) Simrick, N. J.; Bieberle-Hütter, A.; Ryll, T. M.; Kilner, J. A.; Atkin-son, A.; Rupp, J. L. M. An investigation of the oxygen reduction reaction mechanism of $\mathrm{La}_{0.6} \mathrm{Sr}_{0.4} \mathrm{Co}_{0.2} \mathrm{Fe}_{0.8} \mathrm{O}_{3}$ using patterned thin films. Solid State Ion. 2012, 206, 7-16. 